\title{
Impact of finite temperatures on the transport properties of Gd from first principles
}

\author{
K. Chadova, ${ }^{1, *}$ S. Mankovsky, ${ }^{1}$ J. Minár, ${ }^{1,2}$ and H. Ebert ${ }^{1}$ \\ ${ }^{1}$ Department Chemie, Ludwig-Maximilian-University Munich, Butenandtstrasse 5-13, 81377 Munich, Germany \\ ${ }^{2}$ New Technologies-Research Center, University of West Bohemia, Univerzitni 8, 30614 Pilsen, Czech Republic
} (Received 15 September 2016; revised manuscript received 9 February 2017; published 7 March 2017)

\begin{abstract}
Finite-temperature effects have a pronounced impact on the transport properties of solids. In magnetic systems, besides the scattering of conduction electrons by impurities and phonons, an additional scattering source coming from the magnetic degrees of freedom must be taken into account. A first-principle scheme which treats all these scattering effects on equal footing was recently suggested within the framework of the multiple scattering formalism. Employing the alloy analogy model treated by means of the CPA, thermal lattice vibrations and spin fluctuations are effectively taken into account. In the present work the temperature dependence of the longitudinal resistivity and the anomalous Hall effect in the strongly correlated metal Gd is considered. The comparison with experiments demonstrates that the proposed numerical scheme does provide an adequate description of the electronic transport at finite temperatures.
\end{abstract}

DOI: 10.1103/PhysRevB.95.125109

\section{INTRODUCTION}

Rare-earth elements may exhibit both ferromagnetic or antiferromagnetic order in certain temperature regimes. Nowadays, it is commonly accepted that Gd, having the hcp structure, possesses a simple ferromagnetic (FM) order up to its Curie temperature $T_{c}$. However, in early experimental studies a helical magnetic structure was observed in polycrystalline Gd in the temperature range between 210 and $290 \mathrm{~K}[1]$. Such a helical spin configuration is easily destroyed by a weak magnetic field [1], leading to a collinear magnetic structure in the system. This means that only in the absence of an applied magnetic fields can this type of antiferromagnetism be observed. Recent experiments on single crystals of Gd did not reveal any anomalies in the low-field magnetization curves and confirm that Gd has a normal ferromagnetic structure up to its Curie temperature $[2,3]$. The Curie temperature determined experimentally was found to be $289 \mathrm{~K}$ with a saturated magnetic moment of $7.12 \mu_{B}$ [4]. In another experimental study the Curie temperature was determined to be $293.2 \mathrm{~K}$ with an absolute saturation moment of $7.55 \mu_{B}$ [5]. Although $\mathrm{Gd}$ behaves like a simple ferromagnet it has nevertheless a rather complex temperature dependence of its magnetization: as the temperature decreases to 230 a spin-reorientation occurs from the magnetization parallel to the $c$ axis to the magnetization tilted by $30^{\circ}$ with respect to the $c$ axis, reaching its maximum tilt angle of $60^{\circ}$ at around $T=180 \mathrm{~K}$ [2]. Such a behavior is quite demanding concerning an adequate theoretical description. Therefore, in the present work the direction of the magnetization is taken along the $c$ axis unless it is mentioned otherwise.

It is well established that the magnetism in Gd is dominated by $f$ electrons with a magnetic moment of $7 \mu_{B}$ due to half filling of the highly localized $4 f$ states. The observed excessive magnetic moment is attributed to the valence $5 d 6 \mathrm{sp}$ band exhibiting spin polarization due to the strong exchange field created by the $4 f$ electrons [6], as is extensively discussed in the literature [7-11]. In particular, these discussions concern

\footnotetext{
*kchpc@cup.uni-muenchen.de
}

the finite-temperature behavior of the magnetic moment of the valence electrons [12] observed experimentally. In earlier discussions it has been suggested to treat these on the basis of the Stoner model [13]. Recent investigations by experiment $[9,14,15]$ as well as theory $[10,11,16]$ based on firstprinciples calculations clearly demonstrate the finite exchange spitting of valence states above the Curie temperature despite the vanishing total magnetization, which implies a much more complicated picture of interactions than provided by the simple Stoner model.

The rather different origin of the spin magnetic moment for the $f$ and $5 d 6 s p$ electrons leads also to a different dynamical behavior characterized in general by a different magnetization dissipation rate. This would imply separate spin dynamics equations for $f$ and $5 d 6 s p$ spin magnetic moments coupled via the exchange interactions, as was considered in particular in Gilbert damping calculations by Seib and Fähnle [17]. The authors, however, point out that the common equation for all types of spin moments can be used in the limit of slow magnetization dynamics [18], which also allows us to use a common Gilbert damping parameter calculated within the adiabatic approximation.

It is well known that, in magnetic systems, the electrical resistivity is caused by electron scattering by various magnetic inhomogeneities in addition to the electron-phonon scattering as well as scattering by impurities and other structural defects. The latter contribution is responsible for the so-called residual resistivity observed in the zero-temperature limit. The resistivity part due to the phonon mechanism shows usually a $T^{5}$ behavior at low temperatures and varies linearly with $T$ above the Debye temperature $T_{D}$. This behavior can be described on the $a b$ initio level and corresponding studies on transition metals [19] lead in general to good agreement with experimental data. In the present study not only the linear dependence was obtained in the temperature region $T>T_{D}$ but it was found also well below $T_{D}$. A theoretical description of the resistivity caused by thermal spin-fluctuation effects was first given on the basis of the $s$ - $d$ (in rare earth $d$ - $f$ ) model Hamiltonian [20-22]. This approach suggests a $T^{2}$ dependence in the low-temperature limit and an almost constant resistivity above the Curie temperature. In the intermediate-temperature 
regime the $T$ dependence of the resistivity is expected to be rather complex. Recent $a b$ initio calculations of the paramagnetic spin-disorder resistivity for a number of transition metals and their alloys as well as rare-earth metals are based on two alternative approaches: the disordered local moment approach using the coherent-potential approximation (CPA) formalism and averaging the Landauer-Büttiker conductance of a supercell over the random noncollinear spin-disorder configurations, with both leading in general to good agreement with experimental values $[10,23]$. However, for a quantitative description of the temperature-dependent electrical resistivity from first principles one needs to combine the influence of lattice vibrations and spin fluctuations, which is a nontrivial task. Therefore, certain approximations are required to reach this goal.

During the last years, the anomalous Hall effect (AHE) and its dependence on the temperature attracts also much attention. In the case of $\mathrm{Gd}$, a number of theoretical investigations have been performed to explain the unexpectedly large AHE observed experimentally [24]. Previously, these studies were performed on a model level. An earlier description of the AHE of Gd was based on the uniform electron gas model accounting for spin-orbit coupling effects leading, in turn, to an asymmetry in the scattering process (skew-scattering mechanism) [25]. However, due to the high localization the electrons giving rise to the magnetic moment are unable to participate in conduction; therefore this model is not appropriate to describe the AHE in rare-earth systems. The model developed by Kondo [26] was based on the $s-d(s-f)$ interaction leading to a scattering of the conduction electrons by the thermally induced spin moment tilting. In this model the necessary asymmetry is due to the intrinsic spin-orbit coupling of the $f$ electrons. Therefore, the Hamiltonian describing the interaction of the conduction and the localized electrons is valid when the orbital angular moment of localized electrons remains unquenched. This is not the case for $\mathrm{Gd}$ and therefore it cannot be used to describe the AHE in this metal. Another model which eliminated the above-mentioned constraint was developed by Maranzana [27] and is based on Kondo's model. In this model the skew-scattering mechanism originates from the interaction between the localized spin moment and the orbital momentum of the conduction electron.

Within the discussed models the large AHE in Gd was ascribed solely to the skew-scattering contribution. Another scattering mechanism, the so-called side-jump mechanism, first introduced by Berger [28,29], was accounted within a model suggested by Fert [30]. It was demonstrated, particularly for $\mathrm{Gd}$, that the side-jump contribution is equally important as the skew-scattering mechanism and should be taken into account as well.

In this paper, we discuss the impact of finite temperatures, taking into account thermal lattice vibrations and spin fluctuations, on the transport properties in Gd from first principles by making use of the alloy analogy model [31].

\section{COMPUTATIONAL DETAILS}

The electronic structure calculations are based on the Korringa-Kohn-Rostoker (KKR) Green's function method [32] implemented in the fully relativistic spin- polarized Munich SPR-KKR package with angular-momentum cutoff $l_{\max }=4$. A full four-component Dirac formalism is employed to describe the electronic structure within KohnSham-Dirac density functional theory [33]. For spd electrons the local density approximation was used with the parametrization given by Vosko et al. [34]. To treat the highly correlated $4 f$ states the local spin-density approximation $+U$ $(\mathrm{LSDA}+U)$ method was used with the double counting part of the LSDA $+U$ functional evaluated within the so-called atomic limit expression [35]. The temperature effects are treated within the alloy analogy scheme based on the CPA alloy theory [36-38], and assuming a frozen potential for the atoms [31]. For the description of the magnetic spin fluctuations the temperature-dependent magnetization data were taken from experiment [5]. The calculation of the transport properties of Gd is based on the Kubo-Středa formalism, with the corresponding expression for the conductivity given by

$$
\begin{aligned}
\sigma_{\mu \nu}= & \frac{\hbar}{4 \pi N \Omega} \operatorname{Tr}\left\langle\hat{j}_{\mu}\left(G^{+}-G^{-}\right) \hat{j}_{\nu} G^{-}-\hat{j}_{\mu} G^{+} \hat{j}_{\nu}\left(G^{+}-G^{-}\right)\right\rangle_{\mathrm{c}} \\
& +\frac{|e|}{4 \pi i N \Omega} \operatorname{Tr}\left\langle\left(G^{+}-G^{-}\right)\left(\hat{r}_{\mu} \hat{j}_{\nu}-\hat{r}_{\nu} \hat{j}_{\mu}\right)\right\rangle_{\mathrm{c}}
\end{aligned}
$$

with the relativistic current operator $\hat{\mathbf{j}}=-|e| c \alpha$ and the electronic retarded and advanced Green's functions $G^{ \pm}$evaluated at the Fermi energy $E_{F}$ by means of the relativistic multiple scattering or KKR formalism [32]. The angular brackets denote a configurational average which here is carried out using the coherent-potential approximation (CPA) which takes into account the so-called vertex corrections (VCs) [37]. In the last equation, $N$ is the number of sites and $\Omega$ is the volume of the unit cell. As was justified by previous work [39] the second term in the Eq. (1) has been omitted.

The Gilbert damping parameter [40,41] was calculated within the linear-response theory using the Kubo-Greenwoodlike equation:

$$
\alpha_{\mu \nu}=-\frac{\hbar \gamma}{\pi M_{s}} \operatorname{Tr}\left\langle\hat{T}_{\mu} \operatorname{Im} G^{+} \hat{T}_{\nu} \operatorname{Im} G^{+}\right\rangle_{\mathrm{c}},
$$

where $M_{s}$ is the saturation magnetization, $\gamma$ is the gyromagnetic ratio, and $\hat{T}_{\mu}$ is the torque operator [41].

\section{RESULTS}

\section{A. Electronic structure}

The electronic structure of $\mathrm{Gd}$ has been calculated by using the experimental lattice parameters $a=3.629 \AA$, $c / a=1.597$. As was mentioned above, the $4 f$ electrons have been treated as the valence electrons with correlations described within the LSDA $+U$ scheme with the Coulomb parameter $U=6 \mathrm{eV}$ and the exchange parameter $J=0.9 \mathrm{eV}$.

The spin magnetic moment obtained in the calculations for $T=0 \mathrm{~K}$ equals to $7.63 \mu_{B}$ and accordingly is in a good agreement with the experimental saturated magnetic moment of $7.55 \mu_{B}$ per atom [5]. The dominating contribution of $7 \mu_{B}$ is associated with the $f$ electrons, while the excessive spin magnetic moment of $0.63 \mu_{B}$ is a result of the exchange splitting for the $5 d 6 s 6 p$ electrons due to a strong exchange field produced by the $f$ electrons, as was discussed previously $[6,11,12]$. The persistence or vanishing of the exchange splitting with 

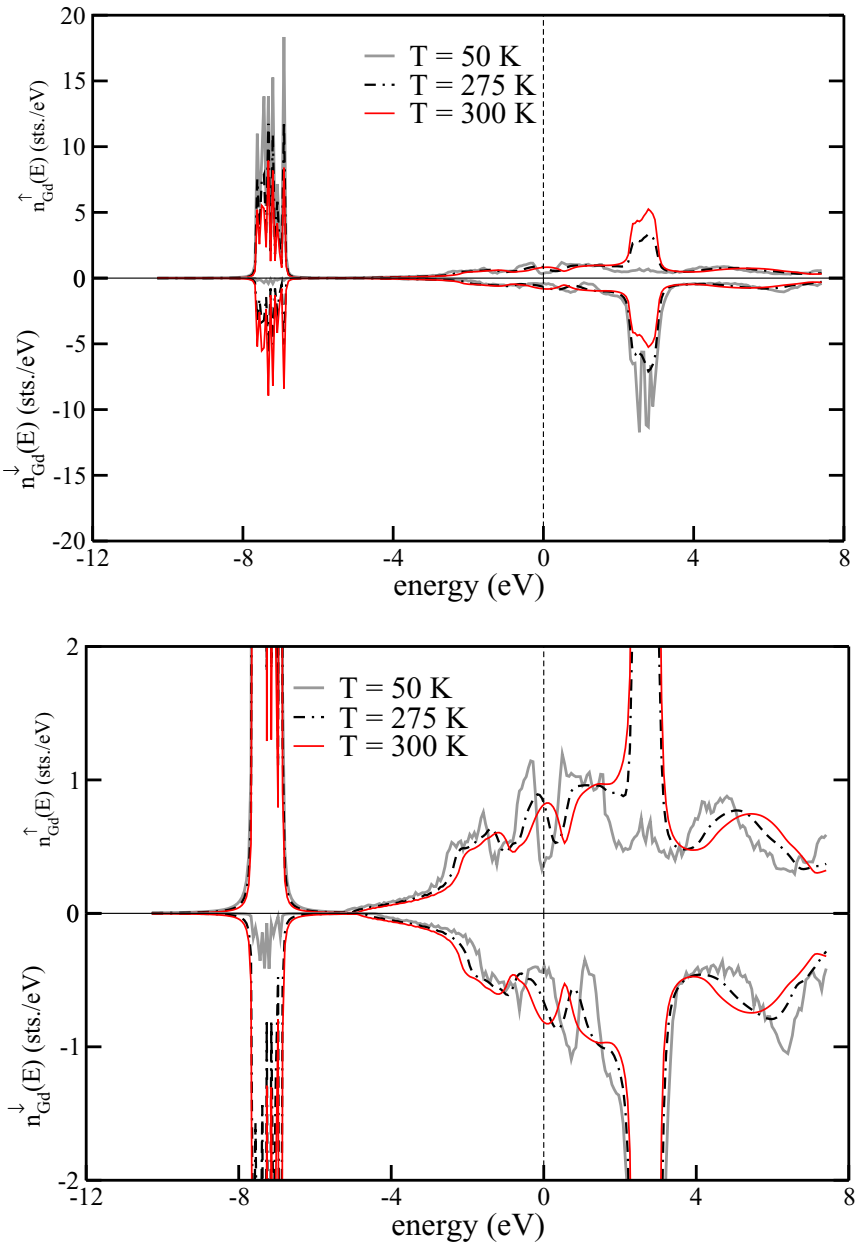

FIG. 1. Spin-resolved DOS of Gd for various temperatures. Bottom panel shows magnified area.

increasing temperature is a matter of debate both in theory and experiment. Several experimental reports indicate that it collapses approaching the Curie temperature [7], while others demonstrate that the exchange splitting persists even in the paramagnetic state $[9,42]$. The spin-resolved total density of states (DOS) calculated in the global frame of reference with the quantization axis along the average magnetization at finite temperatures is represented in Fig. 1. Obviously, a temperature increase results in changes of the majority and minority spin DOS due to the spin mixing caused by the thermal spin fluctuations. This leads to the same DOS for both spin directions at $T>T_{c}$. The energy positions of the $f$ states are almost unchanged in the whole temperature region. However, the exchange splitting of the spin-up and spin-down $5 d 6 s 6 p$ states (having the main contribution to the DOS at the energies around $E_{F}$ ) decreases (as it depends on the average magnetization of the system) with increasing temperature. In particular, this results in an increase of the DOS at the Fermi level in the paramagnetic state.

\section{B. Electrical resistivity}

One of the central transport properties of metallic systems is their electrical resistivity. The experimentally measured
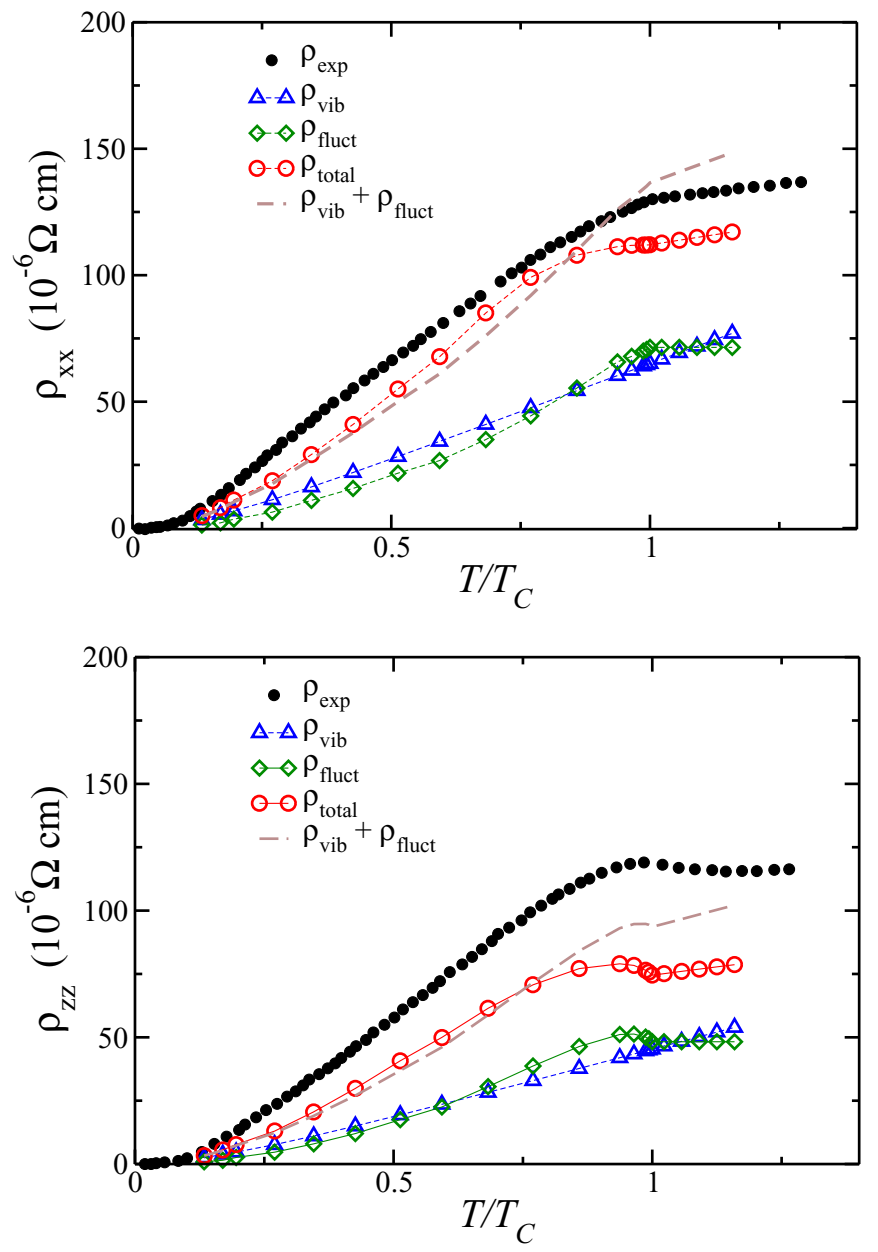

FIG. 2. Temperature-dependent electrical resistivity: (top) inplane, (bottom) out-of-plane components. Black solid circles show experimental results [5], empty blue triangles show only thermal lattice vibrations, empty green diamonds show only spin fluctuations, empty red circles show total resistivity including both effects simultaneously, and brown dashed line corresponds to the sum of individual contributions.

temperature-dependent resistivity of Gd exhibits an anisotropy with different magnitudes along the hexagonal axis $\left(\rho_{z z}\right)$ and in the basal plane $\left(\rho_{x x}\right)$ [5] (see Fig. 2). Both $\rho(T)$ curves are characterized by an abrupt slope change close to the Curie temperature.

In addition to the total $\rho(T)$ values, we investigated its temperature dependence caused only by lattice vibrations (vib) or only by magnetic fluctuations (fluct), which appear to be of comparable magnitude. From this one has to conclude that these sources of the temperature-dependent resistivity are additive only in the case of weak disorder (low temperatures), which does not hold when approaching the Curie temperature (strong disorder) [43]. In this regime they must be taken into account simultaneously, since only then the overall behavior of the resistivity curves agrees well with experiment. This allows us to conclude that the maximum of the experimental $\rho_{z z}$ (close to the Curie temperature) is not a result of short-range magnetic order as was suggested in earlier literature [5], since the present calculations are based on the single-site CPA. The present 


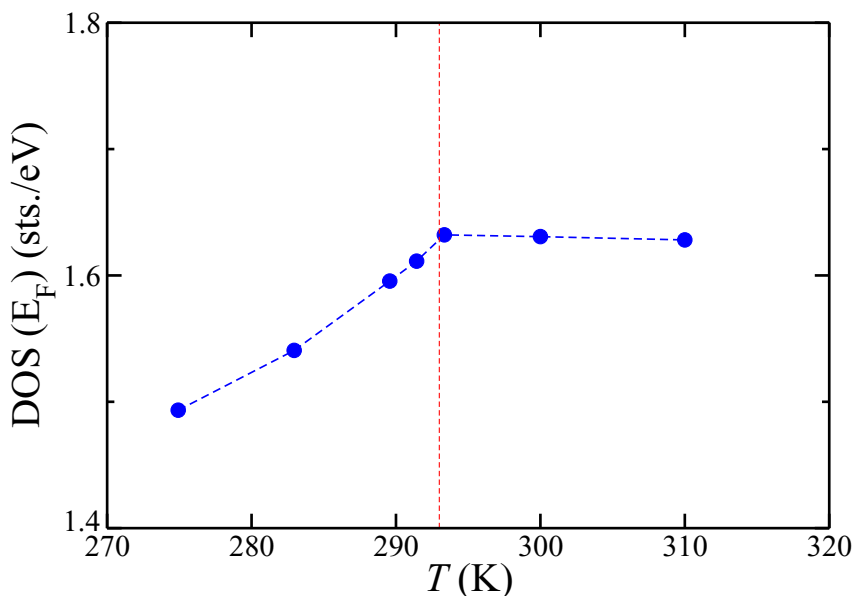

FIG. 3. Total DOS at the Fermi level depending on the temperature.

results suggest its origin as a combination of two competitive mechanisms. On the one hand, thermally induced disorder grows, leading to a resistivity increase and, on the other hand, the effective DOS around $E_{F}$ relevant for the conductivity increases with increasing $T<T_{c}$ (Fig. 3), which effectively reduces the resistivity.

While the calculated resistivities agree with the experiment rather well, there is a quantitative underestimation (see Fig. 2). This can have various sources. One could be the so-called "frozen-potential" approximation used in the present calculations. This approach allows us to account for the most significant contributions to the resistivity, but it neglects the changes in the local magnetic moments with increasing temperature. Nevertheless, as the Gd local magnetic moment is rather robust and does not depend essentially on the temperature, this approximation seems to be well justified. A second reason, which is more crucial for $\rho_{\mathrm{zz}}$, might be the neglect of the anisotropy of the thermal atomic displacements. A third source for discrepancy may be the use of the single-site approximation by the CPA, which neglects the coherent scattering or interference effects which might show up in multiple scattering.

\section{Anomalous Hall effect}

As was already mentioned, Gd shows a rather large AHE, which is well described within a model that accounts at the same time for skew-scattering and side-jump mechanisms [30]. However, within this model only electron scattering by thermally induced spin fluctuations is discussed, while the contribution from the electron-phonon mechanism is completely neglected. Within the present calculations both contributions are taken into account. The resulting total anomalous Hall resistivity can be seen in Fig. 4 (top panel) in comparison with experimental results (for polycrystalline samples as well as single crystals) and the theoretical result obtained on the basis of model calculations by Fert [30]. One can see that the anomalous Hall resistivity shows a pronounced temperature dependence: the resistivity increases from zero at $T=0 \mathrm{~K}$ to a maximum value just below the Curie temperature and then drops to zero as the magnetization vanishes with further
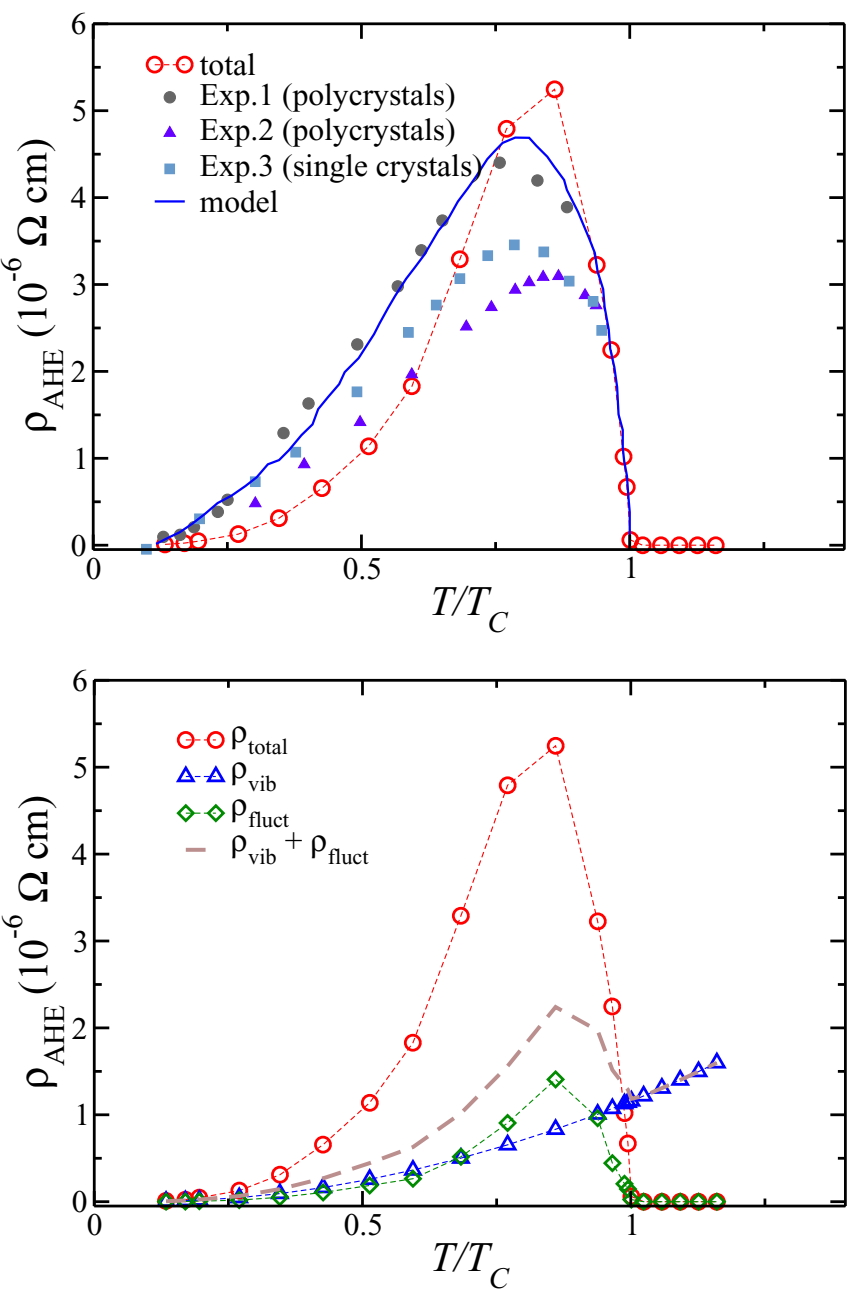

FIG. 4. Anomalous Hall resistivity depending on the temperature: Top panel compares with experimental results (Exp.1 [44], Exp.2 [44], Exp.3 [45,46]) and results from model calculations [30]. Bottom panel shows individual contributions. Empty blue triangles show only thermal lattice vibrations, empty green diamonds show only spin fluctuations, empty red circles show total resistivity including both effects simultaneously, brown dashed line corresponds to the sum of individual contributions.

increasing temperature. Overall there is a qualitative and quantitative agreement of our first-principles results with experiment as well as with the model calculations. In Fig. 4 (bottom panel) the individual contributions arising from the scattering by the lattice vibrations and spin fluctuations are shown. One can see that both mechanisms provide contributions nearly of the same order of magnitude. The qualitative behavior of the total AHR is determined by the scattering due to spin disorder, while the contribution due to lattice vibrations shows, as expected, a monotonic increase with temperature. It is interesting to compare the sum of the individual contributions with the total AHR. From Fig. 4 (bottom panel) one can see that the total AHR significantly exceeds the sum of these contributions. Therefore for the correct description of the total AHR it is necessary to account simultaneously for the combination of scattering due to the thermal lattice vibrations and spin fluctuations. 


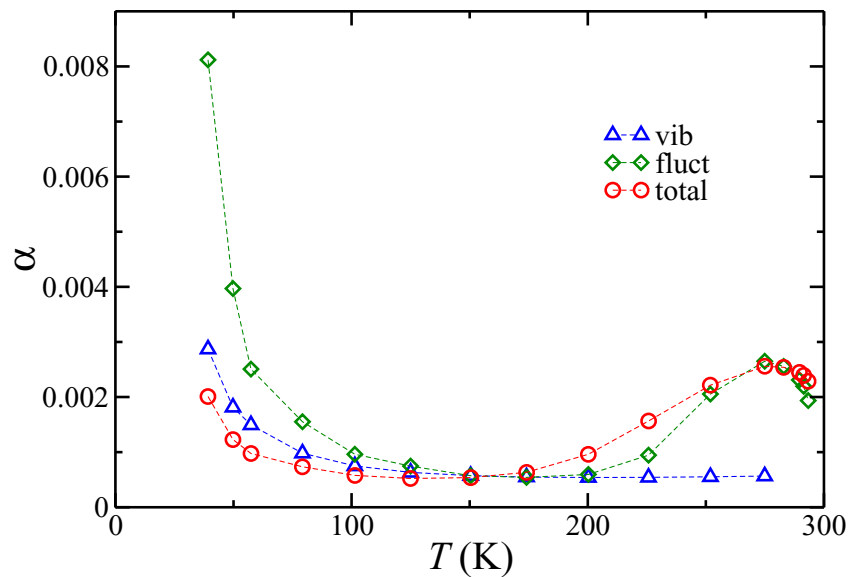

FIG. 5. Gilbert damping parameter in Gd represented as a function of temperature. Empty blue triangles show only thermal lattice vibrations, empty green diamonds show only spin fluctuations, empty red circles show total resistivity including both effects simultaneously.

Comparing the calculated anomalous Hall resistivity with experimental data, one notices that the discrepancy is more pronounced at low temperatures and nearly nonexistent as we approach $T_{\mathrm{C}}$ (see Fig. 4). On one hand, the spread of experimental data is rather large. It would be helpful to have more reliable experimental data to better reveal any systematics in the discrepancies. On the other hand, the discrepancies are connected, of course, to the approximations used in the calculations, which were discussed in the previous section.

\section{Gilbert damping}

Nowadays, much attention is payed to the ultrafast magnetization dynamics in various materials, including $\mathrm{Gd}$ as an important example of rare-earth materials. At the same time, in case of $\mathrm{Gd}$ there is a lack of studies, both theoretical and experimental, particularly dealing with the dissipation channels in the slow magnetization dynamics regime. Our work is meant to fill this gap on the theory side.

In the present work, the Gilbert damping parameter for $\mathrm{Gd}$ has been calculated in the limit of slow magnetization dynamics [18]. It describes the magnetization dissipation for the whole system, accounting for $f$-like and $5 d 6 s p$-like spin magnetic moments characterized by their slow simultaneous coherent motion. The corresponding results of calculations of the Gilbert damping as a function of temperature up to the Curie temperature are shown in Fig. 5. The separate contributions due to thermal lattice vibrations and spin fluctuations are shown together with the curve accounting for both sources simultaneously. One can see a monotonic decrease of the Gilbert damping due to electron-phonon scattering with rising temperature. On the other hand, the curve representing the effect of the electron scattering due to thermal spin fluctuations exhibits a decrease in the low-temperature region due to the dominating breathing Fermi-surface dissipation mechanism, while above $150 \mathrm{~K}$ the increase of the Gilbert damping is determined by the increase of thermal magnetic disorder leading to magnetization dynamics due to electron scattering events accompanied by spin-flip electron transitions. However, approaching the Curie temperature, the Gilbert damping reaches a maximum at $275 \mathrm{~K}$ with a following decrease up to the Curie temperature. This behavior correlates with the temperature-dependent behavior of the resistivity $\rho_{z z}(T)$ and can be associated with the decrease of probability of spin-flip scattering of transport electrons caused by a modification of the electronic structure discussed above. A similar nonmonotonic behavior has been found for the temperature dependence of the total Gilbert damping.

\section{CONCLUSIONS}

In summary, we have studied the transport properties in the highly correlated system Gd from first principles. The electronelectron correlation effects were approximately accounted for by using the LSDA $+U$ approach, resulting in an adequate description of the electronic structure. In turn, it enables a proper physical description of the transport properties. In this contribution we discussed the impact of finite temperatures (including the impact of thermal lattice vibrations and spin fluctuations) on the electrical resistivity as well as on the anomalous Hall resistivity. The applied approach based on the single-site CPA describing thermal lattice vibrations and spin fluctuations allows us to analyze individual contributions to the longitudinal and transverse resistivities arising due to these mechanisms. In both cases it turned out that, in order to obtain reasonable agreement with experimental data, it is necessary to account for a combination of the contributions connected with the phonon scattering and scattering by spin disorder because the simple sum of these contributions, especially for the AHR, significantly deviates from experiment. In the case of the longitudinal resistivity a slight anisotropy was observed which is in agreement with experimental results. For the outof-plane resistivity a small experimentally detected maximum in the vicinity of the Curie temperature was fully reproduced. The emergence of this maximum according to experimental findings was attributed so far to the magnetic short-range-order effect. However, in the present calculations such an ordering was completely neglected because the distribution of the spin magnetic moments are considered absolutely random. Accordingly, the origin of this maximum is solely due to spin disorder.

In case of the AHR a small anisotropy was observed as well. The calculated temperature-dependent AHR with magnetization pointing along the $c$ axis agrees surprisingly well with the experimental data. The maximum occurred just below the Curie temperature and the further abrupt drop is well reproduced.

\section{ACKNOWLEDGMENTS}

This work was financially supported by the Deutsche Forschungsgemeinschaft (DFG) via SFB 689 and FOR1346 (DMFT). The authors would like to thank L. Szunyogh, L. Oroszlány, and S. Chadov for fruitful discussions. 
[1] K. P. Belov and A. V. Ped'ko, Sov. Phys. JETP 15, 62 (1962).

[2] K. Knöpfle and L. M. Sandratskii, Phys. Rev. B 63, 014411 (2000).

[3] G. Will, R. Nathans, and H. A. Alperin, J. Appl. Phys. 35, 1045 (1964).

[4] J. F. Elliott, S. Legvold, and F. H. Spedding, Phys. Rev. 91, 28 (1953).

[5] H. E. Nigh, S. Legvold, and F. H. Spedding, Phys. Rev. 132, 1092 (1963).

[6] L. W. Roeland, G. J. Cock, F. A. Muller, A. C. Moleman, K. A. McEwen, R. G. Jordan, and D. W. Jones, J. Phys. F: Met. Phys. 5, L233 (1975).

[7] B. Kim, A. B. Andrews, J. L. Erskine, K. J. Kim, and B. N. Harmon, Phys. Rev. Lett. 68, 1931 (1992).

[8] E. Weschke, C. Schüssler-Langeheine, R. Meier, A. V. Fedorov, K. Starke, F. Hübinger, and G. Kaindl, Phys. Rev. Lett. 77, 3415 (1996).

[9] K. Maiti, M. C. Malagoli, A. Dallmeyer, and C. Carbone, Phys. Rev. Lett. 88, 167205 (2002).

[10] J. K. Glasbrenner, K. D. Belashchenko, J. Kudrnovský, V. Drchal, S. Khmelevskyi, and I. Turek, Phys. Rev. B 85, 214405 (2012).

[11] L. Oroszlány, A. Deák, E. Simon, S. Khmelevskyi, and L. Szunyogh, Phys. Rev. Lett. 115, 096402 (2015).

[12] S. Khmelevskyi, I. Turek, and P. Mohn, Phys. Rev. B 70, 132401 (2004).

[13] M. Donath, B. Gubanka, and F. Passek, Phys. Rev. Lett. 77, 5138 (1996).

[14] D. Li, J. Pearson, S. D. Bader, D. N. McIlroy, C. Waldfried, and P. A. Dowben, Phys. Rev. B 51, 13895 (1995).

[15] A. V. Fedorov, T. Valla, F. Liu, P. D. Johnson, M. Weinert, and P. B. Allen, Phys. Rev. B 65, 212409 (2002).

[16] L. M. Sandratskii, Phys. Rev. B 90, 184406 (2014).

[17] J. Seib and M. Fähnle, Phys. Rev. B 82, 064401 (2010).

[18] J. Seib, D. Steiauf, and M. Fähnle, Phys. Rev. B 79, 064419 (2009).

[19] S. Y. Savrasov and D. Y. Savrasov, Phys. Rev. B 54, 16487 (1996).

[20] T. Kasuya, Prog. Theor. Phys. 16, 58 (1956).

[21] T. Kasuya, Prog. Theor. Phys. 22, 227 (1959).

[22] P. de Gennes and J. Friedel, J. Phys. Chem. Solids 4, 71 (1958).
[23] J. Kudrnovský, V. Drchal, I. Turek, S. Khmelevskyi, J. K. Glasbrenner, and K. D. Belashchenko, Phys. Rev. B 86, 144423 (2012).

[24] M. Christen, B. Giovannini, and J. Sierro, Phys. Rev. B 20, 4624 (1979).

[25] R. Karplus and J. M. Luttinger, Phys. Rev. 95, 1154 (1954).

[26] J. Kondo, Prog. Theor. Phys. 27, 772 (1962).

[27] F. E. Maranzana, Phys. Rev. 160, 421 (1967).

[28] L. Berger, Phys. Rev. B 2, 4559 (1970).

[29] L. Berger, Phys. Rev. B 5, 1862 (1972).

[30] R. Asomoza, A. Fert, and R. Reich, J. Less-Common Met. 90, 177 (1983).

[31] H. Ebert, S. Mankovsky, K. Chadova, S. Polesya, J. Minár, and D. Ködderitzsch, Phys. Rev. B 91, 165132 (2015).

[32] H. Ebert, D. Ködderitzsch, and J. Minár, Rep. Prog. Phys. 74, 096501 (2011).

[33] E. Engel and R. M. Dreizler, Density Functional Theory - An Advanced Course (Springer, Berlin, 2011).

[34] S. H. Vosko, L. Wilk, and M. Nusair, Can. J. Phys. 58, 1200 (1980).

[35] M. T. Czyżyk and G. A. Sawatzky, Phys. Rev. B 49, 14211 (1994).

[36] B. Velický, Phys. Rev. 184, 614 (1969).

[37] W. H. Butler, Phys. Rev. B 31, 3260 (1985).

[38] I. Turek, J. Kudrnovský, V. Drchal, L. Szunyogh, and P. Weinberger, Phys. Rev. B 65, 125101 (2002).

[39] T. Naito, D. S. Hirashima, and H. Kontani, Phys. Rev. B 81, 195111 (2010).

[40] A. Brataas, Y. Tserkovnyak, and G. E. W. Bauer, Phys. Rev. Lett. 101, 037207 (2008).

[41] H. Ebert, S. Mankovsky, D. Ködderitzsch, and P. J. Kelly, Phys. Rev. Lett. 107, 066603 (2011).

[42] D. Li, J. Zhang, P. A. Dowben, and M. Onellion, Phys. Rev. B 45, 7272 (1992).

[43] J. K. Glasbrenner, B. S. Pujari, and K. D. Belashchenko, Phys. Rev. B 89, 174408 (2014).

[44] N. Babushkina, Sov. Phys. Solid State 7, 2450 (1966).

[45] R. S. Lee and S. Legvold, Phys. Rev. 162, 431 (1967).

[46] N. V. Volkenshtein, I. K. Grigorova, and G. V. Fedorov, Sov. Phys. JETP 23, 1003 (1966). 